\title{
Estrutura de uma floresta tropical dez anos após exploração de madeira em Moju, Pará ${ }^{1}$
}

\author{
Fernando Cristóvam da Silva Jardim ${ }^{2 *}$, Larissa Corrêa Lopes Quadros ${ }^{3}$
}

10.1590/0034-737X201663040001

\section{RESUMO}

Estudos das alterações florísticas e estruturais em áreas de exploração florestal permitem avaliar os impactos e fornecem informações básicas para o manejo florestal racional. Nesse contexto, as dinâmicas florística e estrutural foram avaliadas em uma floresta tropical, dez anos após sua exploração madeireira. O experimento foi executado em 200 ha do Campo Experimental da Embrapa Amazônia Oriental, em Moju, PA, onde foi feita a exploração madeireira. No entorno de nove clareiras selecionadas foram instaladas faixas de $10 \mathrm{~m}$ x $50 \mathrm{~m}$, divididas em parcelas quadradas de 10 $m$ de lado (1 a 5), onde foram inventariadas as plantas com DAP $\geq 5 \mathrm{~cm}$. Nas parcelas 1,3 e 5 e no centro da clareira foram instaladas subparcelas de $2 \mathrm{~m}$ x $2 \mathrm{~m}$, onde foram medidos os indivíduos com altura $<10 \mathrm{~cm}$ e DAP $<5 \mathrm{~cm}$. O monitoramento da floresta abrangeu um período de nove anos e meio, entre março de 1998 e outubro de 2007. A análise estrutural foi feita com base nos parâmetros de abundância, frequência, dominância, regeneração natural, posição sociológica e índice de valor de importância ampliado (IVIA), sendo comparadas as estruturas da floresta dos anos de 2007 e 1998. Dez anos após a exploração, a alta heterogeneidade foi mantida e aumentou no povoamento mais jovem, graças ao ingresso de espécies com forte demanda por luz. Entretanto, a composição florística e a estrutura da floresta manejada ainda mostram grande importância de espécies que, em florestas não perturbadas, não teriam grande expressão em termos de IVIA.

Palavras-chave: análise estrutural; dinâmica florística; dinâmica estrutural; floresta tropical; manejo florestal; monitoramento.

\section{ABSTRACT}

\section{Structure of a tropical rainforest 10 years after selective logging in Moju-Pará}

Studies of the floristic and structural dynamics in forest exploitation areas allow to assess the impacts and provide basic information for the rational forest management. In this context, the floristic and structural dynamics were evaluated in a rainforest, ten years after its logging. The experiment was performed in 200 ha of the Experimental Field of Embrapa Eastern Amazon, in Moju - PA, where logging was done. Around the nine gaps selected, $10 \mathrm{~m}$ x $50 \mathrm{~m}$ tracks were installed, divided into square plots of $10 \mathrm{~m}$ side (1-5), where the plants with DBH $\geq 5 \mathrm{~cm}$ were inventoried. In plots 1,3 and 5 and in the gap center subplots of $2 \mathrm{~m} \mathrm{x} 2 \mathrm{~m}$ were installed where individuals with height $<10 \mathrm{~cm}$ and DBH $<5 \mathrm{~cm}$ were measured. The forest monitoring covered a period of 9.5 years, between March 1998 and October 2007. The structural analysis was based on the parameters of abundance, frequency, dominance, natural regeneration, sociological position and extended importance value index (EIVI) and compared the forest structures in 2007 and 1998. Ten years after logging, the high heterogeneity was maintained and increased in the younger population, due to the entry of species with strong demand for light. However, the floristic composition and structure of the managed forest still show great importance of species that, in undisturbed forests, would not have great expression in terms of EIVI.

Key words: structural analysis; floristic dynamics; structural dynamics; tropical rainforest; forest management; monitoring.

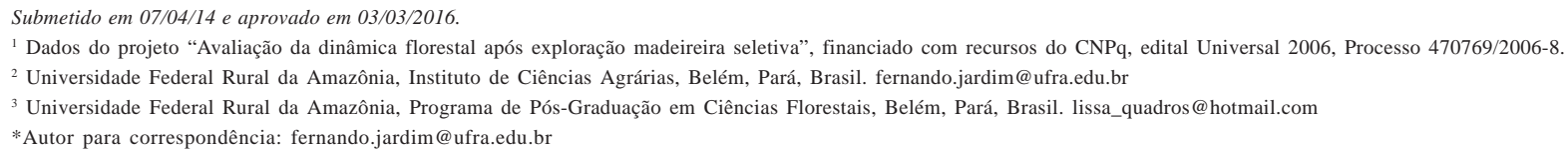




\section{INTRODUÇÃO}

A maioria das florestas tropicais nativas da Amazônia tem sido explorada de forma não sustentável, o que implica em perda da cobertura florestal e da diversidade de espécies, antes mesmo que se tenha o conhecimento dessa riqueza natural. Isso tem levado a sociedade a questionar os modelos de utilização dos recursos da floresta

São inúmeras as exigências da humanidade para a conservação desse rico ecossistema. Várias medidas já foram tomadas para se chegar a essa meta e uma delas é a adoção de planos de manejo que visam a utilizar a floresta de forma racional (Souza et al., 2006).

O conhecimento da composição florística e da estrutura da floresta permite o planejamento e o estabelecimento de sistemas de manejo com produção sustentável e condução da floresta a uma estrutura balanceada, bem como a práticas silviculturais adequadas (Souza et al., 2006). Uma forma de se obter dados sobre estrutura, a composição florística e a dinâmica de florestas é a partir do monitoramento em parcelas permanentes. Diversos trabalhos têm sido realizados por meio desse método e geraram informações importantes sobre o comportamento das espécies florestais e arbóreas dentro das florestas (Mory \& Jardim, 2001; Nemer \& Jardim, 2004; Jardim \& Vasconcelos, 2006; Jardim \& Soares, 2010), assim como sobre as florestas como um todo (Jardim \& Hosokawa, 1986/87; Jardim \& Silva, 2003; Francez et al., 2007), gerando subsídios para decisões silviculturais no manejo.

A partir desses dados podem-se fazer deduções sobre a origem, as características ecológicas e sinecológicas, a dinâmica e as tendências de desenvolvimento da floresta, sendo esses elementos básicos para o planejamento do manejo florestal (Jardim et al., 2008). Por outro lado, os desenvolvimentos florístico e estrutural de uma floresta que sofreu exploração madeireira é ainda pouco conhecido, mas é imprescindível para avaliar a sustentabilidade ecológica do manejo florestal.

A análise estrutural, conforme proposta por Finol (1971) e também aplicada por Jardim \& Hosokawa (1986/ 87), aborda a estrutura horizontal, na qual se avaliam a abundância, a frequência e a dominância das espécies, assim como a estrutura vertical, que inclui os parâmetros posição sociológica e regeneração natural.

A estrutura horizontal, conquanto importante em estudos de vegetação, não permite a perfeita caracterização da hierarquia das espécies em termos de importância ecológica, pois nada informa acerca da sua estrutura diamétrica. Tal informação é essencial para caracterizar o grau de estabilidade ecológica e de sustentabilidade dessas espécies no manejo florestal, o que foi inserido na análise estrutural por meio dos parâmetros da estrutura vertical, proposta por Finol (1971).
As projeções de ciclo de corte nos planos de manejo de florestas naturais devem levar em consideração o recrutamento, a mortalidade e o crescimento das espécies, dos quais depende todo o processo dinâmico de recomposição e reestruturação da floresta. Todavia, pouquíssimos estudos têm sido feitos no sentido da caracterização das alterações da estrutura total de florestas impactadas por atividades de manejo florestal (Francez et al., 2007; Francez et al., 2009; Mendes et al., 2013).

O objetivo deste estudo foi avaliar os impactos da exploração madeireira seletiva sobre a composição florística e a estrutura de uma floresta tropical, após dez anos de monitoramento.

\section{MATERIAL E MÉTODOS}

A área de estudos localiza-se no Campo Experimental da Embrapa Amazônia Oriental, km 30 da Rodovia PA 150, no município de Moju, Pará, com 1059 ha, situado entre as latitudes $2^{\circ} 07^{\prime} 30^{\prime \prime} \mathrm{Se} 2^{\circ} 12^{\prime} 06^{\prime \prime} \mathrm{S}$ e longitudes $48^{\circ} 46^{\prime} 57^{\prime \prime}$ $\mathrm{O}$ e $48^{\circ} 48^{\prime} 30^{\prime \prime} \mathrm{O}$.

O clima do município é do tipo mesotérmico e úmido, em que a temperatura média anual é elevada e varia entre 25 e $27^{\circ} \mathrm{C}$. Os solos são representados pela classe Latossolos Amarelos e Argissolos Amarelos típicos (Santos et al., 1985).

A cobertura vegetal da região é composta por uma floresta tropical densa de terra firme, constituída por árvores de grande porte, com altura do dossel na faixa de 25 a 35 m (Serrão et al., 2003).

Dentro da Estação Experimental da Embrapa, foi selecionada uma área de 200 ha, a qual sofreu exploração florestal seletiva em 1997, a partir de uma parceria entre a Embrapa e uma empresa madeireira. Nessa área, foram selecionadas nove clareiras provenientes da exploração, com tamanho mínimo de $231 \mathrm{~m}^{2}$, e no entorno marcadas parcelas amostrais (Figura 1). Faixas de $10 \mathrm{~m}$ x $50 \mathrm{~m}$ foram instaladas, começando na bordadura da clareira para dentro da floresta, nas direções Norte, Sul, Leste e Oeste, portanto, quatro faixas por clareira. Cada faixa foi dividida em parcelas quadradas de $10 \mathrm{~m}$ de lado, nas quais foram medidos os indivíduos com DAP $\geq 5 \mathrm{~cm}$. Dentro das parcelas localizadas na borda, a 20 e a $40 \mathrm{~m}$ da borda, foram instaladas subparcelas quadradas de $2 \mathrm{~m}$ de lado, onde foram medidos todos os indivíduos com altura total $\geq 10$ $\mathrm{cm}$ e DAP $<5 \mathrm{~cm}$.

No início do estudo, os espécimes desconhecidos foram identificados por nomes vulgares e tiveram material vegetativo coletado para a determinação a partir da comparação nos Herbários IAN da Embrapa Amazônia Oriental e do Museu Emílio Goeldi. O sistema de classificação botânica utilizado foi o de "Angiosperm Phylogeny 
Group" (APG III, 2009) e a nomenclatura botânica conferida com o banco de dados eletrônico disponibilizado pelo Missouri Botanical Garden (Mobot, 2010).

A partir da primeira medição, a cada três meses e sempre logo após o final de cada estação do ano, foram feitas medições, as quais começaram em junho de 1998 e terminaram em junho de 2001. Em 2007 foram feitas mais duas medições, totalizando 15 medições. Para este estudo, foram utilizados os dados da $1^{\mathrm{a}}$ e da $15^{\mathrm{a}}$ medição, portanto, de um intervalo de nove anos e meio de monitoramento.

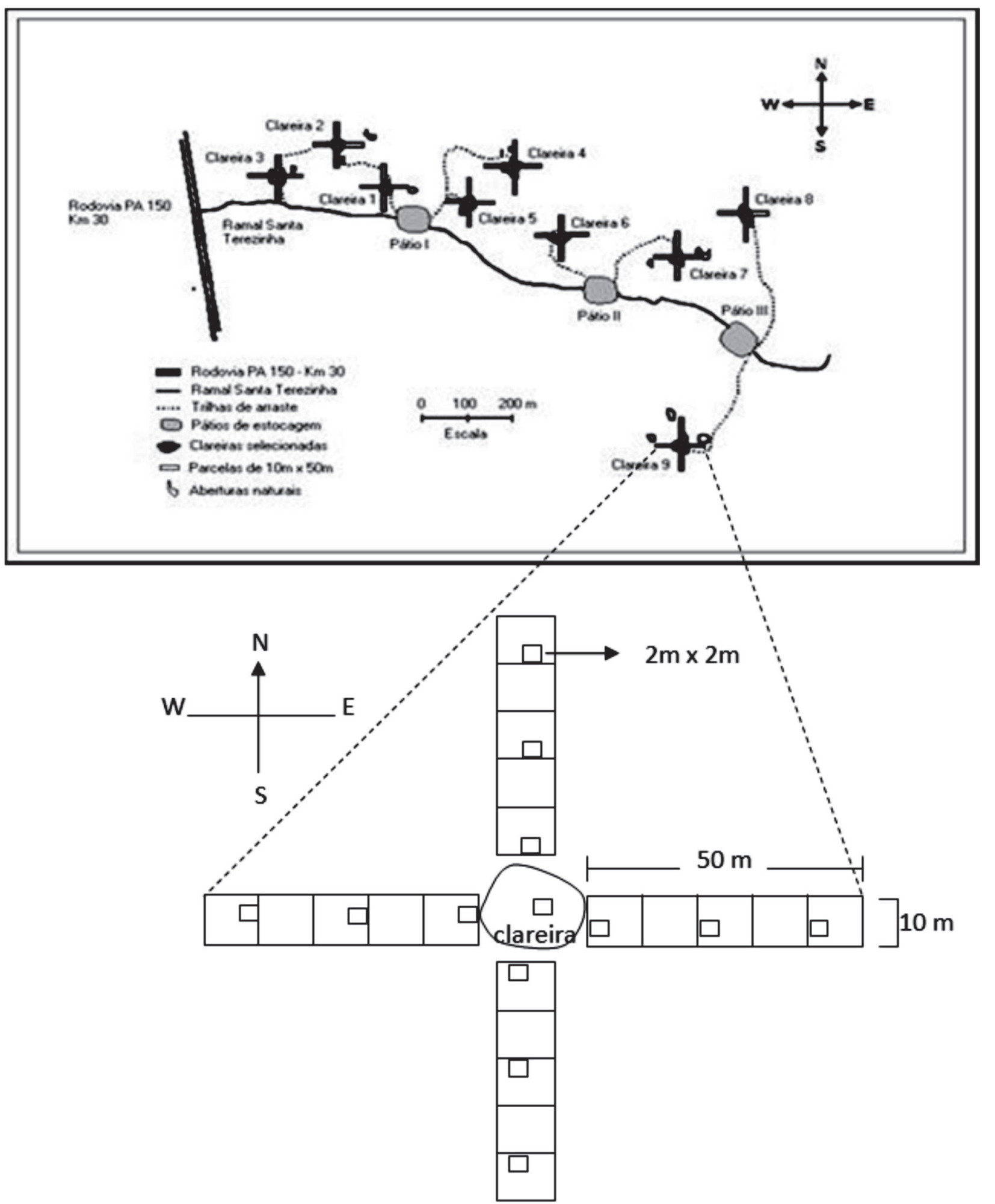

Figura 1: Desenho esquemático da distribuição espacial das amostras nas clareiras no Campo Experimental da Embrapa Amazônia Oriental, Moju, Pará. 
A composição florística do ecossistema florestal estudado foi analisada por meio do Quociente de Mistura de Jentsch (QM), que permite ter ideia geral da composição florística da floresta, sendo usado como um fator para medir o grau de heterogeneidade florística da área (Jardim \& Hosokawa, 1986/1987). O QM foi calculado pela seguinte expressão:

\section{$Q M=\underline{\text { número de espécies }}$ número de indivíduos}

A análise estrutural foi feita segundo a metodologia apresentada em Jardim \& Hosokawa (1986/1987), que preconiza a divisão da floresta em estrutura horizontal e vertical e o cálculo do Índice de Valor de Importância Ampliado (IVIA\%), dado pela média aritmética dos valores relativos dos parâmetros das estruturas horizontal e vertical. Para o cálculo dos parâmetros Abundância, Dominância, Frequência e Posição Sociológica foram considerados os indivíduos com DAP $\geq 20 \mathrm{~cm}$, enquanto aqueles com altura total maior ou igual a $5 \mathrm{~cm}$ e DAP menor que $20 \mathrm{~cm}$ foram considerados para o cálculo do parâmetro Regeneração Natural.

Considerou-se para análise da variação estrutural somente os valores de IVIA das 40 espécies mais importantes nas duas medições (1998 e 2007), embora se faça uso dos parâmetros isolados para explicar essas variações.

\section{RESULTADOS E DISCUSSÃO}

\section{Análise das variações florísticas}

Considerando as duas medições, a floresta inventariada apresentou uma composição florística heterogênea, com 314 espécies, 146 gêneros e 64 famílias. A primeira medição, realizada em 1998, apresentou 270 espécies, 136 gêneros e 62 famílias. As alterações da floresta quanto ao recrutamento e mortalidade constataram-se, ao final dos nove anos e meio de monitoramento, 302 espécies, 144 gêneros e 63 famílias.

As famílias mais comuns entre as 314 espécies da área inventariada foram: Mimosaceae (com 25 espécies), Fabaceae (12 espécies), Sapotaceae (12 espécies); Chrysobalanaceae (10 espécies), Lauraceae (10 espécies) e Moraceae (10 espécies); Burseraceae (9 espécies) e Caesalpiniaceae ( 9 espécies); Annonaceae (8 espécies), Lecythidaceae (8 espécies) e Vochysiaceae (8 espécies); Clusiaceae (7 espécies) e Euphorbiaceae (7 espécies). Essas famílias juntas foram responsáveis por $43 \%$ do total de espécies da área estudada.

Os gêneros mais comuns entre as 314 espécies que ocorreram na área foram: Inga (8 espécies); Pouteria (7 espécies) e Protium (7 espécies); Eschweilera (6 espécies); Brosimum, Cordia, Licania e Ocotea (5 espécies cada um) e Cecropia, Pithecellobium e Rinorea (4 espécies cada um). Esses gêneros perfazem 7,5\% do total de gêneros que ocorreram na área estudada.

Das 314 espécies, 259 foram comuns aos dois períodos de monitoramento, sendo que 86 ocorreram somente na regeneração natural, 24 na população adulta e 112 estão presentes tanto na regeneração como na população adulta. As 259 espécies comuns estão distribuídas em 61 famílias botânicas, com 134 gêneros. Entre essas 61 famílias botânicas, as que apresentaram maior diversidade de espécies foram: Mimosaceae (com 21 espécies), Fabaceae (11 espécies), Sapotaceae (10 espécies), Lauraceae, (9 espécies), Vochysiaceae, Moraceae, Lecythidaceae e Burseraceae (8 espécies cada um), Caesalpinoideae (7 espécies), Euphorbiaceae, Clusiaceae e Cecropiaceae (6 espécies cada um). Essas famílias juntas foram responsáveis por $41,7 \%$ das espécies comuns.

Entre os 134 gêneros, os mais representativos foram: Inga com 7 espécies, Pouteria (6), Brosimum, Eschweilera e Licania (5 espécies cada um), Cecropia, Cordia e Ocotea (4 espécies cada um), Casearia, Vochysia, Rinorea, Qualea, Pithecellobium, Parkia e Lecythis (3 espécies cada um). Esses gêneros juntos são responsáveis por $23,5 \%$ das espécies comuns nas duas medições.

Nessa floresta, estudos antes e imediatamente após a exploração mostraram que havia o predomínio das espécies Rinorea guianensis Aubl. (acariquarana), Eschweilera coriacea (A. DC.) Mori (matamatá branco), Lecythis idatimon Aubl. (ripeiro), Protium pilosum (Cuatz.) Daly (breu) e Vouacapoua americana Aubl. (acapu) (Costa et al., 1998; Jardim \& Vasconcelos, 2006).

Os resultados do Quociente de Mistura de Jentsch (Tabela 1), embora muito próximos nas duas medições, tanto para o povoamento com DAP < $5 \mathrm{~cm}(1: 26 \mathrm{em} 1998 \mathrm{e}$ $1: 25$ em 2007) como para aquele maior ou igual a $5 \mathrm{~cm}$ de DAP (1:10 em 1998 e 1:11 em 2007), indicam que a regeneração natural se tornou mais heterogênea e o povoamento adulto mais homogêneo. Todavia, a floresta como um todo manteve-se a heterogeneidade, sugerindo que a exploração florestal não afetou a composição florística da área. Esses valores indicam que a floresta de Moju é mais homogênea do que a floresta da região de Manaus, cujo Quociente de Mistura de Jentsch foi de 1:8 (Jardim \& Hosokawa, 1986/1987).

Os resultados deste estudo indicam que o sub-bosque da floresta estudada apresenta caráter mais homogêneo que o dossel, o que é corroborado por outros estudos na Amazônia, em que se demonstra um aumento da heterogeneidade na estrutura da floresta do subbosque para o dossel (Jardim \& Hosokawa, 1986/1987; Lima Filho et al., 2002). Esses valores são atribuídos a algumas espécies típicas do sub-bosque, cuja densidade é muito elevada, como Monotagma secundum 
(cantan), com 598 indivíduos e Calathea aberrans (cauaçu), com 489 indivíduos, no ano de 2007, as quais impõem um caráter mais homogêneo a esse estrato da floresta.

Alguns resultados (Swaine \& Hall, 1983; Mendes et al., 2012) têm demonstrado que, logo após a perturbação, a diversidade florística da vegetação sucessional é maior que a da floresta madura. Portanto, o manejo florestal com base na regeneração natural é, antes de tudo, uma garantia de manutenção e até mesmo de aumento na diversidade biológica.

\section{Variações na importância ecológica das espécies}

As espécies Eschweilera coriaceae (matamatá branco), Rinorea guianensis (acariquarana) e Lecythis idatimon (ripeiro) foram as mais importantes, ecologicamente, nesse período de nove anos, pois nas duas medições, realizadas em 1998 e 2007, as três permaneceram em $1^{\circ}, 2^{\circ}$ e $3^{\circ}$ lugares, respectivamente (Tabela 2). As espécies Eschweilera coriaceae e Rinorea guianensis, apesar de terem mantido as primeiras posições, tiveram os valores de IVIA\% reduzidos por causa da queda dos valores de seus parâmetros estruturais. Lecythis idatimon, ao contrário, teve um aumento de 0,92 no seu IVIA\%, graças ao aumento de seus parâmetros estruturais, principalmente da dominância, causado pelo ingresso de cinco indivíduos em contrapartida à baixa mortalidade e ao aumento do CAP da população.

Um estudo realizado por Souza et al (2006), em uma floresta ombrófila densa de terra firme, não explorada, mostrou que Lecythis idatimon ocupou o primeiro lugar na hierarquia de importância, graças aos elevados valores de dominância e densidade. As espécies Eschweilera coriacea e Rinorea guianensis também entraram na classificação das espécies de maior importância relativa, o que demonstra o alto grau de recuperação da floresta que sofreu uma exploração florestal.

Eschweilera blanchetiana (matamatá preto) e Vouacapoua americana (acapu), que estavam na $4^{\mathrm{a}}$ e $5^{\mathrm{a}}$ posições no ano de 1998, respectivamente, inverteram suas posições na medição realizada em 2007 (Tabela 2). Eschweilera blanchetiana teve o seu IVIA\% reduzido, por causa da queda de todos os parâmetros estruturais, ocasionada pela intensa mortalidade dos indivíduos aci- ma de $10 \mathrm{~cm}$ de altura (regeneração natural) e pela morte de dois indivíduos com CAPs de $130 \mathrm{~cm}$ e $122 \mathrm{~cm}$. Vouacapoua americana mostrou aumento em todos os valores de seus parâmetros. Essa espécie apresentou um rápido crescimento em circunferência, no período de nove anos, com indivíduos que tiveram aumentos de CAP de 7 $\mathrm{cm}, 10 \mathrm{~cm} \mathrm{e} 18 \mathrm{~cm}$, por exemplo.

Pouteria sp. (abiu arrepiado), Cecropia purpurascens (embaúba vermelha), Manilkara huberi (maçaranduba), Pouteria macrophylla (abiurana) e Chrysophyllum prieurii (abiu casca fina), que estavam na $6^{\mathrm{a}}, 8^{\mathrm{a}}, 10^{\mathrm{a}}, 14^{\mathrm{a}} \mathrm{e}$ $17^{\mathrm{a}}$ posições, respectivamente, tiveram uma pequena queda nas suas posições por causa da redução dos valores de IVIA\% (Tabela 2).

Pouteria sp. caiu uma posição, indo do $6^{\circ}$ para o $7^{\circ}$ lugar, com redução de 0,28 no IVIA\%. Essa pequena diferença é explicada pelo fato de todos os parâmetros, com exceção da dominância e da regeneração natural, terem diminuído, principalmente o parâmetro abundância, por causa de grande número de indivíduos que morreram. O mesmo aconteceu com Cecropia purpurascens, que reduziu apenas uma posição, indo do $8^{\circ}$ para o $9^{\circ}$ lugar na classificação das 40 espécies de maior IVIA \%.

Manilkara huberi desceu da $10^{\mathrm{a}}$ para a $13^{\mathrm{a}}$ posição na hierarquia do povoamento, o que ocorreu principalmente pela queda significativa do parâmetro posição sociológica, com uma redução de 0,88 no seu valor.

Pouteria macrophylla e Chrysophyllum prieurii caíram uma posição cada, indo da $14^{\mathrm{a}}$ para a $15^{\mathrm{a}}$ posição e da $17^{\mathrm{a}}$ para a $18^{\mathrm{a}}$ posição hierárquica, respectivamente. Pouteria macrophylla teve redução de todos os seus parâmetros, com exceção da dominância, fazendo que essa espécie caísse apenas uma posição. A intensa mortalidade dessa espécie permitiu essa redução na posição. Chrysophyllum prieurii sofreu redução de todos os seus parâmetros, permitindo que o seu IVIA\% se reduzisse de 0,167 .

Pouteria guianensis (abiu vermelho), Cecropia obtusa (embaubão) e Tachygalia mymercophilla (tachi preto) subiram de posição. Pouteria guianensis teve um aumento de 0,052 no seu IVIA\%, devido ao aumen-

Tabela 1: Quociente de Mistura de Jentsch no ano de 1998 e 2007, de uma floresta explorada em Moju, PA

\begin{tabular}{|c|c|c|c|c|c|c|}
\hline & \multicolumn{2}{|c|}{$\mathbf{R N}$} & \multicolumn{2}{|c|}{ PA } & \multicolumn{2}{|c|}{ Total } \\
\hline & 1998 & 2007 & 1998 & 2007 & 1998 & 2007 \\
\hline Espécies & 230 & 266 & 175 & 192 & 270 & 302 \\
\hline Indivíduos & 6049 & 6595 & 1827 & 2086 & 7876 & 8681 \\
\hline QM & $1: 26$ & $1: 25$ & $1: 10$ & $1: 11$ & $1: 29$ & $1: 29$ \\
\hline
\end{tabular}

RN = Regeneração Natural; PA = Povoamento Adulto; QM= Quociente de Mistura de Jentsch 
to dos parâmetros abundância, frequência e posição sociológica, apesar da alta mortalidade dos indivíduos acima de $10 \mathrm{~cm}$ de altura, fazendo que o parâmetro regeneração natural tivesse um decréscimo de 0,29 no seu valor. Cecropia obtusa subiu 1,338 no valor de seu IVIA\%. Esse fato é explicado pelo rápido crescimento em diâmetro que essa espécie apresentou, como o aumento no CAP de $83 \mathrm{~cm}$ para $114,4 \mathrm{~cm}$, de $91 \mathrm{~cm}$ para $108 \mathrm{~cm}$, e de $81 \mathrm{~cm}$ para $101 \mathrm{~cm}$ em um intervalo de tempo de nove anos. Tachygalia mymercophilla obte- ve um aumento de 0,573 no seu IVIA\%, graças principalmente ao incremento de CAP de oito indivíduos dessa espécie, variando entre $25 \mathrm{~cm}$ até $135 \mathrm{~cm}$ de aumento da circunferência.

Protium tenuifolium (breu preto), Licania sclerophylla (caripé), Bauhinia cf. coronata (escada de jabuti) e Sterculia pruriens (axixá) subiram na classificação das 40 espécies de maior IVIA\%, em um período de nove anos.

Tabela 2: Ordenamento das espécies pelo Índice de Valor de Importância Ampliado (IVIA), nas estruturas da floresta tropical de terra firme da Estação Experimental da Embrapa Amazônia Oriental, Moju, PA, em 1998 e 2007

\begin{tabular}{|c|c|c|c|c|}
\hline Espécie & $\begin{array}{c}\text { IVIA \% } \\
1998\end{array}$ & Hierarquia & $\begin{array}{c}\text { IVIA \% } \\
2007\end{array}$ & Espécie \\
\hline Eschweilera coriaceae (A. DC.) Mori & 9,470 & $1^{\circ}$ & 8,714 & Eschweilera coriaceae (A. DC.) Mori \\
\hline Rinorea guianensis Aubl. & 8,228 & $2^{\circ}$ & 7,919 & Rinorea guianensis Aubl. \\
\hline Lecythis idatimon Aubl. & 6,398 & $3^{\circ}$ & 7,318 & Lecythis idatimon Aubl. \\
\hline Eschweilera blanchetiana (Berg) Miers & 5,013 & $4^{\circ}$ & 4,295 & Vouacapoua americana Aubl. \\
\hline Vouacapoua americana Aubl. & 3,955 & $5^{\circ}$ & 3,442 & Eschweilera blanchetiana (Berg) Miers \\
\hline Pouteria sp. & 2,691 & $6^{\circ}$ & 3,224 & Cecropia obtussa Trécu L. \\
\hline Trattinickia burseraefolia (Mart.) Wild. & 2,151 & $7^{\circ}$ & 2,411 & Pouteria sp. \\
\hline Cecropia purpurascens C. C. Berg. & 2,070 & $8^{\circ}$ & 2,393 & Tachigalia myrmecophylla Ducke \\
\hline Cecropia obtussa Trécu L. & 1,886 & $9^{\circ}$ & 1,984 & Cecropia purpurascens C. C. Berg. \\
\hline Manilkara huberi (Ducke) Chevalier & 1,824 & $10^{\circ}$ & 1,888 & Licania sclerophylla (Mart ex Hook) Fristch. \\
\hline Tachigalia myrmecophylla Ducke & 1,820 & $11^{\circ}$ & 1,842 & Pouteria guianensis Aubl. \\
\hline Pouteria guianensis Aubl. & 1,790 & $12^{\circ}$ & 1,742 & Sterculia pruriens (Aubl.) K. Schum. \\
\hline Lecythis pisonis Cambers & 1,744 & $13^{\circ}$ & 1,562 & Manilkara huberi (Ducke) Chevalier \\
\hline Pouteria macrophylla (Lam) Eyma & 1,510 & $14^{\mathrm{o}}$ & 1,378 & Trattinickia burseraefolia (Mart.) Wild. \\
\hline Inga thibaudiana DC & 1,412 & $15^{\circ}$ & 1,269 & Pouteria macrophylla (Lam) Eyma \\
\hline Sterculia pruriens (Aubl.) K. Schum. & 1,390 & $16^{\circ}$ & 1,243 & Protium tenuifolium (Engl.) Engl. \\
\hline Chrysophyllum prieurii A. DC & 1,388 & $17^{\circ}$ & 1,241 & Apeiba membranacea Spruce ex Benth. \\
\hline Chrysophyllum venezuelanense (Pierre) T. D. & 1,358 & $18^{\circ}$ & 1,221 & Chrysophyllum prieurii A. DC \\
\hline Newtonia suaveolens (Miq.) Brenan. & 1,354 & $19^{\circ}$ & 1,159 & Protium subserratum Engl. \\
\hline Protium subserratum Engl. & 1,256 & $20^{\circ}$ & 1,111 & Inga sp \\
\hline Striphnodendron paniculatum Poepp. \& Endl. & 1,102 & $21^{\circ}$ & 1,101 & Inga thibaudiana DC \\
\hline Protium tenuifolium (Engl.) Engl. & 1,050 & $22^{\circ}$ & 1,023 & Lecythis pisonis Cambers \\
\hline Licania sclerophylla (Mart ex Hook) Fristch. & 1,038 & $23^{\circ}$ & 0,977 & Newtonia suaveolens (Miq.) Brenan. \\
\hline Hynenaea courbaril L. & 0,946 & $24^{\circ}$ & 0,949 & Symphonia globulifera L. F. \\
\hline Protium trifoliolatum Engl. Breu & 0,932 & $25^{\circ}$ & 0,949 & Theobroma subincanum Mart. \\
\hline Virola melinonii (R. Benoist) A. C. Sm. & 0,932 & $26^{\circ}$ & 0,881 & Parkia ulei (Harms) Kuhlm \\
\hline Manilkara paraensis (Huber) Standl. & 0,924 & $27^{\circ}$ & 0,881 & Striphnodendron paniculatum Poepp. \& Endl. \\
\hline Monotagma secundum & 0,916 & $28^{\circ}$ & 0,827 & Cordia bicolor A. DC \\
\hline Apeiba membranacea Spruce ex Benth. & 0,912 & $29^{\circ}$ & 0,783 & Chrysophyllum venezuelanense (Pierre) T. D. \\
\hline Cecropia leucocoma Miquel. & 0,902 & $30^{\circ}$ & 0,751 & Bauhinia $c f$. coronata Benth. \\
\hline Parkia ulei (Harms) Kuhlm & 0,890 & $31^{\circ}$ & 0,751 & Hynenaea courbaril L. \\
\hline Cordia bicolor A. DC & 0,854 & $32^{\circ}$ & 0,745 & Monotagma secundum \\
\hline Ocotea canaliculata (Rich.) Mez. & 0,840 & $33^{\circ}$ & 0,719 & Protium guacayanum Cuatrec. \\
\hline Lecythis lurida (Miers) S. A. Mori & 0,810 & $34^{\circ}$ & 0,717 & Brosimum lactescens (S. Moore) C. C. Berg \\
\hline Caryocar glabrum (Aubl.) Pers. & 0,796 & $35^{\circ}$ & 0,711 & Virola melinonii (R. Benoist) A. C. Sm. \\
\hline Licania micrantha Miq. & 0,774 & $36^{\circ}$ & 0,703 & Rinorea flavescens (Aubl.) Kuntze \\
\hline Micrandra elata Muell. Arg. & 0,762 & $37^{\circ}$ & 0,689 & Eschweilera alba Knuth. \\
\hline Protium guacayanum Cuatrec. & 0,748 & $38^{\circ}$ & 0,677 & Calathea aberrans \\
\hline Qualea paraensis Ducke & 0,740 & $39^{\circ}$ & 0,643 & Anacardium giganteum Loud. Ex. Steud. \\
\hline Bauhinia cf. coronata Benth. & 0,694 & $40^{\circ}$ & 0,637 & Cecropia leucocoma Miquel. \\
\hline
\end{tabular}


Protium tenuifolium subiu da $22^{\mathrm{a}}$ para a $16^{\mathrm{a}}$ posição, com um aumento de 0,193 no IVIA \% . Isso ocorreu graças ao aumento de seus parâmetros abundância, frequência e, principalmente, regeneração natural, com um alto índice de ingresso de indivíduos acima de $10 \mathrm{~cm}$ de altura. Licania sclerophylla subiu da $23^{\mathrm{a}}$ para a $10^{\mathrm{a}}$ posição, com um aumento de 0,85 no valor do IVIA\%. Esse aumento explica-se pelo incremento de seus parâmetros estruturais, com exceção da regeneração natural.

Bauhinia cf. coronata aumentou de posição, apesar de ter tido o seu IVIA\% reduzido. Esse fato decorre do aumento do número de espécies entre as duas medições, passando de 270 espécies, na primeira medição, para 302, na segunda, resultando numa repartição do IVIA por um maior número de espécies, na medição de 2007, diminuindo a magnitude desse parâmetro por espécie.

Sterculia pruriens subiu quatro posições na hierarquia do povoamento, indo da $16^{\mathrm{a}}$ para a $12^{\mathrm{a}}$ posição. Essa espécie teve um aumento de 0,352 no seu IVIA\% ocasionado pelo aumento de todos os seus parâmetros, com exceção da regeneração natural. $\mathrm{O}$ principal fator foi o parâmetro dominância, no qual houve um aumento de 0,73 no seu valor, ocasionado pelo aumento do CAP de sete indivíduos, variando entre $12 \mathrm{~cm}$ e $36 \mathrm{~cm}$, em um período de nove anos.

Monotagma secundum (cantan), Trattinickia burseraefolia (almesclão), Hymenaea courbaril (jatobá), Stryphnodendron paniculatum (tachi pitomba) e Lecythis pisonis (sapucaia) desceram de posição nesse período de nove anos.

Monotagma secundum foi da $28^{\mathrm{a}}$ para a $32^{\mathrm{a}}$ posição, descendo quatro posições na classificação hierárquica por causa do parâmetro regeneração natural ter-se reduzido de 0,85 , ocasionado pela morte de muitos indivíduos. As espécies Trattinickia burseraefolia e Hymenaea courbaril reduziram sete posições cada, indo da $7^{\mathrm{a}}$ para a $14^{\mathrm{a}}$ posição, e da $24^{\mathrm{a}}$ para a $31^{\mathrm{a}}$ posição, respectivamente. Ambas as espécies tiveram todos os parâmetros estruturais reduzidos. T. burseraefolia mostrou uma alta mortalidade dos indivíduos acima de $10 \mathrm{~cm}$ de altura, quando, de 219 indivíduos, contabilizados na primeira medição, restaram apenas 62 indivíduos, em 2007 e, também, por causa da morte de um indivíduo de $200 \mathrm{~cm}$ de CAP. H. courbaril reduziu de posição porque todos os indivíduos da regeneração natural morreram.

Stryphnodendron paniculatum desceu seis posições, ocupando a $27^{\mathrm{a}}$ posição, na medição realizada em 2007, e Lecythis pisonis caiu nove posições, ficando na $35^{\mathrm{a}}$ posição, em decorrência da redução de todos os seus parâmetros estruturais, com exceção da regeneração na- tural, que se manteve para S. paniculatum. Em contrapartida, morreu o único indivíduo de $L$.pisonis e ingressou um indivíduo em 2007.

Cecropia leucocoma (embaúba branca) desceu da $30^{\text {a }}$ para a $40^{a}$ posição, ocupando o último lugar das quarenta espécies de maior IVIA\%, tendo redução de 0,265. Todos os parâmetros estruturais dessa espécie foram reduzidos por morte de cinco indivíduos da população adulta, de um total de 11 na primeira medição, restando apenas seis indivíduos na medição de 2007. Outro fator foi a mortalidade de todos os indivíduos da regeneração natural, dos quais restou apenas um indivíduo com $11 \mathrm{~cm}$ de altura (Tabela 2).

Oito espécies saíram da classificação das 40 espécies de maior IVIA\%. São elas: Protium trifoliolatum (breu), Manilkara paraensis (maparajuba), Ocotea canaliculata (louro branco), Lecythis lurida (jarana), Caryocar glabrum (piquiarana), Licania micrantha (cariperana), Micrandra elata (seringarana) e Qualea paraensis (mandioqueira escamosa). A saída dessas espécies foi ocupada por Inga sp. (ingá), Symphonia globulifera (anani), Theobroma subincanum (cupuí), Brosimum lactescens (janitá), Rinorea flavescens (canela de jacamim), Eschweilera alba (matamatá jiboia), Calathea aberrans (cauaçu) e Anacardium giganteum (cajuaçu).

Protium trifoliolatum estava na $25^{\mathrm{a}}$ posição e saiu da classificação das 40 espécies de maior IVIA\%, por morte de 159 indivíduos que estavam presentes na regeneração natural, no ano de 1998. Manilkara paraensis, Ocotea canaliculata e Lecythis lurida (jarana) caíram de posição por causa da redução de todos os parâmetros estruturais, principalmente regeneração natural, causada pela morte de muitos indivíduos de M. paraensis.

Caryocar glabrum, Licania micrantha, Micrandra elata e Qualea paraensis, que estavam nas $35^{\mathrm{a}}, 36^{\mathrm{a}}, 37^{\mathrm{a}} \mathrm{e}$ $39^{a}$ posições, saíram da classificação das 40 espécies de maior IVIA\%, por causa da redução dos valores de IVIA $\%$. O IVIA de $M$. elata reduziu-se por morte de um indivíduo de $246 \mathrm{~cm}$ de CAP e por morte de indivíduos da regeneração natural.

Inga sp. subiu para o $20^{\circ}$ lugar, com aumento de 0,513 no seu IVIA\%, principalmente graças ao ingresso de nove indivíduos na população adulta. Symphonia globulifera, Theobroma subincanum e Brosimum lactescens subiram por causa do aumento de seus parâmetros estruturais, com exceção da regeneração natural para T. subincanum.

Rinorea flavescens, Eschweilera alba, Calathea aberrans e Anacardium giganteum subiram para as $36^{\mathrm{a}}$, $37^{\mathrm{a}}, 38^{\mathrm{a}}$ e $39^{\mathrm{a}}$ posições, respectivamente (Tabela 2). Rinorea flavescens teve aumento em todos os parâmetros estrutu- 
rais, e isso foi possível graças ao recrutamento de 207 indivíduos nesse período de nove anos. E. alba mostrou aumento nos parâmetros abundância, frequência e dominância. C. aberrans teve um pequeno aumento do IVIA \%, mas, pelo fato dos valores de IVIA\% serem menores em 2007, permitiu-se o ingresso. A. giganteum obteve aumento em todos os parâmetros estruturais graças ao rápido crescimento que essa espécie apresentou.

Ao longo de seis anos de monitoramento em floresta tropical não perturbada, verificou-se que a hierarquia relativa das espécies, em termos de IVIA só foi alterada a partir da vigésima posição (Jardim \& Silva, 2003). Neste estudo, no entanto, os resultados mostram que, após a exploração madeireira, a importância ecológica relativa das espécies sofreu grande alteração, já nas primeiras posições, em termos de IVIA. Isso foi corroborado pelos valores moderados do coeficiente de correlação de Spearman entre os IVIA de 1998 e de 2007, avaliados em p= 0,80, para as 20 espécies mais importantes em termos de IVIA, e em $\mathrm{p}=0,66$, quando avaliado para as 50 espécies mais importantes.

As principais diferenças entre $\mathrm{o}$ ambiente na clareira $\mathrm{e}$ sob o dossel fechado são o aumento da luz e mudanças na sua qualidade, o aumento de temperatura e um déficit hídrico. Pode haver também aumento da disponibilidade de nutrientes, quando plantas mortas são decompostas (Whitmore, 1978). Essas mudanças no meio físico alteram a biocenose, pois mudas estabelecidas morrem por sua sensibilidade à luz, plantas de espécies secundárias aparecem e outras têm seu crescimento maximizado (Jardim et al., 1993).

A interação entre o ambiente alterado das clareiras provocadas pela exploração madeireira e os diferentes grupos ecológicos que compõem a vegetação pode explicar a ascensão, na hierarquia do IVIA, de espécies conhecidamente mais intolerantes à sombra, como Cecropia obtusa, Tachigalia myrmecophylla, Sterculia pruriens e Inga sp., cujo recrutamento e crescimento foram favorecidos ao longo dos dez anos de monitoramento, mas que deverão se estabilizar depois do fechamento das clareiras, como mostraram alguns resultados nessa área de estudo (Nemer \& Jardim, 2004; Jardim \& Vasconcelos, 2006; Jardim \& Soares, 2010; Santos \& Jardim, 2012).

\section{CONCLUSÕES}

Dez anos após a exploração madeireira, a alta heterogeneidade foi mantida, e até aumentou, no sub-bosque da floresta estudada, graças ao ingresso de espécies com forte demanda por luz. Por outro lado, a composição florística e a estrutura da floresta manejada ainda não voltaram ao estado original, pois mostrou-se grande a importância de espécies que em florestas não perturbadas não teriam grande expressão em termos de IVIA.

\section{REFERÊNCIAS}

APG III (2009) An update of the angiosperm phylogeny group classification for the orders and families of flowering plants: APG III. Botanical Journal of the Linnean Society, 161:105121.

Costa DHM, Ferreira CAP, Silva JNM, Lopes JCA \& Carvalho JOP (1998) Potencial madeireiro de 200 hectares de floresta densa no município de Moju, Estado do Pará. Belém, EmbrapaCPATU. 33p. (Documentos, 121).

Finol UH (1971) Nuevos Parámetros a Considerarse en el Análisis Estructural de las Selvas Virgenes Tropicales. Revista Forestal Venezolana, 14:29-42.

Francez LMB, Carvalho JOP \& Jardim FCS (2007) Mudanças ocorridas na composição florística em decorrência da exploração florestal em uma área de floresta de terra firme na região de Paragominas, PA. Acta Amazonica, 37:219-228.

Francez LMB, Carvalho JOP, Jardim FCS, Quanz B \& Pinheiro KAO (2009) Efeito de duas intensidades de colheita de madeira na estrutura de uma floresta natural na região de Paragominas, Pará. Acta Amazonica, 39:851-864.

Jardim FCS \& Hosokawa RT (1986/1987) Estrutura da floresta equatorial úmida da Estação Experimental de Silvicultura Tropical do INPA. Acta Amazonica, 16/17:411-508.

Jardim FCS, Sena JRC \& Miranda IS (2008) Dinâmica e estrutura da vegetação com DAP e" $5 \mathrm{~cm}$ em torno de clareiras da exploração florestal seletiva, em Moju Pará. Revista de Ciências Agrárias, 49:41-52.

Jardim FCS \& Silva GAP (2003) Análise da variação estrutural da floresta equatorial úmida da Estação Experimental de Silvicultura Tropical do Instituto Nacional de Pesquisa da Amazônia INPA, Manaus - AM. Revista de Ciências Agrárias, 39:25-54.

Jardim FCS \& Soares MS (2010) Comportamento de Sterculia pruriens (Aubl.) Schum. em floresta tropical manejada em MojuPA. Acta Amazonica, 40:535-542.

Jardim FCS \& Vasconcelos LMR (2006) Dinâmica da regeneração natural de Rinorea guianensis Aublet, em uma floresta tropical primária explorada seletivamente, Moju(PA). Revista de Ciências Agrárias, 45:121-134.

Jardim FCS, Volpato MML \& Souza AL (1993) Dinâmica de sucessão natural em clareiras de florestas tropicais. Viçosa, SIF. 60p. (Documento, 10).

Lima Filho DA, Revilla J, Coêlho LS, Ramos JF, Santos JL \& Oliveira JG (2002) Regeneração Natural de três hectares de floresta ombrófila densa de terra firme na região do Rio UrucuAM, Brasil. Acta Amazonica, 32:555-569.

Mendes FS, Jardim FCS, Carvalho JOP, Souza DV, Araújo CB, Oliveira MG \& Leal ES (2013) Dinâmica da estrutura da vegetação do sub-bosque sob influência da exploração em uma floresta de terra firme no município de Moju - PA. Ciência Florestal, 23:379-391.

Mendes FS, Jardim FCS, Carvalho JOP, Lima TTS \& Souza DV (2012) Dinâmica da composição florística do sub-bosque em floresta tropical manejada, no município de Moju, estado do Pará, Brasil. Revista de Ciências Agrárias, 55:117-123.

MOBOT (2010) Missouri Botanical Garden. Trópicos Search. Disponível em: <http://www.tropicos.org/Home.aspx >. Acessado em: 15 de junho de 2010 .

Mory AM \& Jardim FCS(2001) Comportamento de Goupia glabra Aubl. (Cupiúba) em diferentes níveis de desbaste por anelamento em florestas naturais. Revista de Ciências Agrárias, 36:55-66. 
Nemer TC \& Jardim FCS (2004) Crescimento diamétrico de uma população de Eschweilera odora (POEPP.) Miers com DAP e' $5 \mathrm{~cm}$ durante três anos em uma floresta tropical de terra-firme manejada, Moju-Pará-Brasil. Revista de Ciências Agrárias, 41:7788 .

Santos CAN \& Jardim FCS (2012) Dinâmica da regeneração natural de Vouacapoua americana com diâmetro $<5 \mathrm{~cm}$, influenciada por clareiras, em Moju, Pará. Floresta, 42:495-508.

Santos PL, Silva JML, Silva BNR, Santos RD \& Rego GS (1985) Levantamento semidetalhado dos solos e avaliação da aptidão agrícola das terras para culturas de dendê e seringueira. Rio de Janeiro, Embrapa/SNLCS. p. 192. (relatório técnico).
Serrão DR, Jardim FCS \& Nemer TC (2003) Sobrevivência de seis espécies florestais em uma área explorada seletivamente no município de Moju, Pará. Revista Cerne, 9:153-163.

Souza DR, Souza AL, Leite HG \& Yared JAG (2006) Análise Estrutural em floresta ombrófila densa de terra firme não explorada, Amazônia Oriental. Revista Árvore, 30:75-87.

Swaine MD \& Hall JB (1983) Early succession on cleared forest land in Ghana. Journal of Ecology, 71:601-627.

Whitmore TC (1978) Gaps in the forest canopy. In: Tomlinson PB \& Zimmerman MH (Eds.) Tropical trees as living systems. London, Cambridge University Press. p. 639-655. 